\title{
ImALeG: A Serious Game for Amazigh Language Learning
}

\author{
https://doi.org/10.3991/ijet.v14i18.10854 \\ Yassine Tazouti $(\bowtie)$ \\ University Ibn Tofail, Kenitra, Morocco \\ yassine.tazouti@gmail.com \\ Siham Boulaknadel \\ Royal Institute of Amazigh Culture, Rabat, Morocco \\ Youssef Fakhri \\ University Ibn Tofail, Kenitra, Morocco
}

\begin{abstract}
At present, serious games are experiencing a growing popularity and popularity, with areas of application that extend not only to education, but also to other sectors such as the military, health and business sectors, among others. Since video games facilitate the learning of complex processes, their associated benefits have been reoriented principally to the educational, training and information processes.

This paper presents "ImALeG" project, a 3D serious game, whose objective is to develop and auto evaluate competencies of Amazigh language learning in a virtual environment. ImALeG is a serious language game designed for all age groups who want to learn Tifinaghe in an interactive way. The game leverages the use of virtual reality developed with Unity 3D game engine to implement immersive learning as well as a multi-agent system to ensure game interactivity.
\end{abstract}

Keywords - Serious games, Educational game, learning game, Language learning, Multi-agent System, Artificial intelligence.

\section{Introduction}

Nowadays the situation of Amazigh language in Morocco having changed, its status reconsidered, being official language besides Arabic. This factor makes Amazigh educating increasingly commons in grade schools, as well as in some Moroccan middle school.

Today, the new generation is expert in using technology. We can say learning Amazigh by gaming would be one of the greatest methods for our target audience. In other hand, serious games can provide a fun learning environment, so the learner can play during his free time in order to enjoy learning the Amazigh language. The greatest fragment is that while the learner is immersed by the game, he is in fact learning. So rather than he pays attention to standard learning, he simply focusses on progress- 
ing in the game (which also needs planned thinking, which can expand more his skills).

Furthermore, learning Amazigh through games could motivate and improve engagement to learn more about the language. This work aims to provide a $3 \mathrm{D}$ environment filled with objects with which the learner can interact in this environment, learners perform exercises that focus on Amazigh Alphabet called "Tifinagh". The game envisioned helping player learn Tifinagh alphabet and expand their verbal skills in a fun and joyful way. The idea of the game is to offer teachers a new interactive tool that might actually speed up the learning progression and at the same time make it further interesting and entertaining.

\section{Related Work}

Many research studies have discovered the potential of serious games and showed that exploiting them as educational tools might be effective. According to $[1,2,3]$, serious games proved to be helpful in the learning field, as they can offer some features that the traditional teaching approaches lack.

In the last decade, the learning language research has been concerned on applying 3D virtual reality environments, identical to Second Life, for languages acquisition. Certainly, this type of environments affords immersive learning and facilitates distance learning. Likewise, these advances could develop learning as a social practice giving students to employ dynamic communication.

A further system entitled SGLL ProjectX system, was made in 2008 at the Dublin Institute of Technology's School of Computing. The ProjectX system places users in a 3D environment with a static camera and permits the users to move from place to place by picking objects in the scene, which the camera changes to focus on. The system is basically a set of mini-games related to a mutual field [7]. The user, for example, had the capability to gather components to make a recipe in a $3 \mathrm{D}$ kitchen environment or carrying out a grocery list in a 3D marketplace environment.

A connected system, known as Middle world Online, was developed in 2010 by Middle-bury College's Interactive Languages division. The system offers the users an online interactive world permitting them absorbed in the language learning environment through coursework, actions, and mini-games [8]. The mini-game places players in charge of waiting tables at a restaurant. The users have the capacity to interact with clients and preserve them completely pleased by identifying orders and in what manner to answer them properly in the target language. Making errors will outcome is an unhappy client and a lower global score.

In the other hand, there are numerous studies that exploit multi-agent systems as an adaptation technique in serious games due to their behavior and active ability to change the global game settings.

Therefore, there are a limited notable language learning projects situated in multiagent systems. One of these projects, which suggest a design architecture of serious online games based on monitoring the characters to follow the development of the player's skills to guarantee a real-time adaptation of the agent's behaviors, and to 
avoid scenario disturbance during adaptation. The project has developed an organizational structure for planning agents to track the storyline evolution [9].

\section{$3 \quad$ Amazigh Language}

\subsection{Historical background of amazighe language}

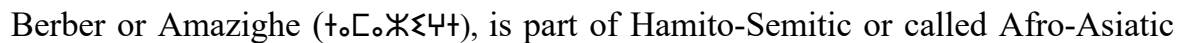
[10]. It is currently presented in a dozen countries ranging from Morocco, with 50\% of the overall population1, to Egypt, passing through Algeria with 25\%, the Tunisia, Mauritania, Libya, Niger and the Mali. In Morocco [15], we distinguish between three major Amazighe dialects: Tarifit in the north, Tamazight in the center, and Tashelhit in the south [16].

\subsection{Amazighe learning approach}

The approach adopted by Moroccan primary school for teaching Amazigh language is inspired from the pedagogy of skills. The textbook designers assume that Amazigh teaching aim to mastering the learner communicative competence [17], mainly oral skill that is based on dialogue and secondarily written skill, both in terms of expression and comprehension. Gradually, once acquired the strategies of the oral, reading and writing, the learner learns cultural competence through activities, these pedagogical approaches seems to be traditional and does not apply to this generation who tend to explore new information technologies that are so far unused in our educational system.

From this point, we have found that serious games will be useful for Amazigh teaching as a pedagogical medium which based on the motivation of learners through an emerging narrative story and on transmission skills using feedbacks through learning by trial and error approach.

\section{Our Approach}

ImALeG is a serious game ongoing project developed by LaRiT lab to help people and students to learn Amazighe language. This scientific project is a first attempt to exploit the 3D virtual reality environment provided by Unity platform to implement immersive and learn-by-doing techniques that have proved to be among the most effective learning strategies in language acquisition. The game provides an environment to learn vocabulary (on some specific topics as furniture, animals, food, colors, shapes) to learners of Amazigh language. In this paper, we have focused on Tifinaghe alphabet learning as a first step witch we consider essential before learning vocabulary.

\footnotetext{
${ }^{1}$ It presents the Amazigh population largest in number.
} 
In what follows, we start by describing the game scenario used for language learning. Then, we describe the features, the game screen and the system architecture.

\subsection{Game scenario}

We imagined the game scene as a village populated with houses, a restaurant, a cinema, supermarket, zoo, forest, each of which representing a game unit corresponding to a particular lexical field to be acquired by the learner. The ImALeG forest is currently the main game unit to learn Tifinaghe alphabet.

A first-person perspective is employed whereby the learner is an avatar that can easily move in the game world. In order to start the game, the learner is prompted to fill an information form corresponding to his age, level of education, native language and sex; in order to define players profile and to store these information's in external database for later retrieval.

Once these options have been set, the learner arrives at the main menu where there are a few other useful settings such as camera and audio options world selection input mode. He chooses the avatar to represent himself in the game scene, and then the player will be redirected to the first level of learning, the tifinaghe world in which the player is in a forest containing multiple animals and multiple 3D Tifinaghe alphabets to collect.

A Non-Player character (NPC) called Ahmed comes to the avatar. He presents the first quest that the player must solve during the scene, the player must follow the NPC instructions which guide him finding Tifinaghe alphabet and teach him each alphabet. The player is able to select any alphabet on the screen with the mouse, which causes the pronunciation of the selected alphabet. Double-clicking an alphabet displays the "Info Menu" screen, providing more details about the alphabet, such as the pronunciation description, translation into the learner's native language and its category either consonants, semi-consonants or vowels and he can listen the spelling of the tifinaghe alphabet.

Once the player collects 10 alphabets that have been added to his inventory, the quest ends with a scene to evaluate these 10 characters found. The tutor agent explains to the learner what to do, the learner through a mini-map witch define the positions of each Tifinaghe alphabets he can fallow a specific path to reach Tifinaghe alphabet. Whenever the learner is approaching a Tifinaghe alphabets from a specified distance, a pronunciation is triggered with information panel. The learner then is prompted to recognize the alphabet and fill it in an inventory table. Once the alphabet is recognized, the learner stores energy in the health bar in order to continue the evaluation process. The next stage of the evaluation is to classify these alphabets in inventory tables according to their types semi-consonant, vowel or consonant. 


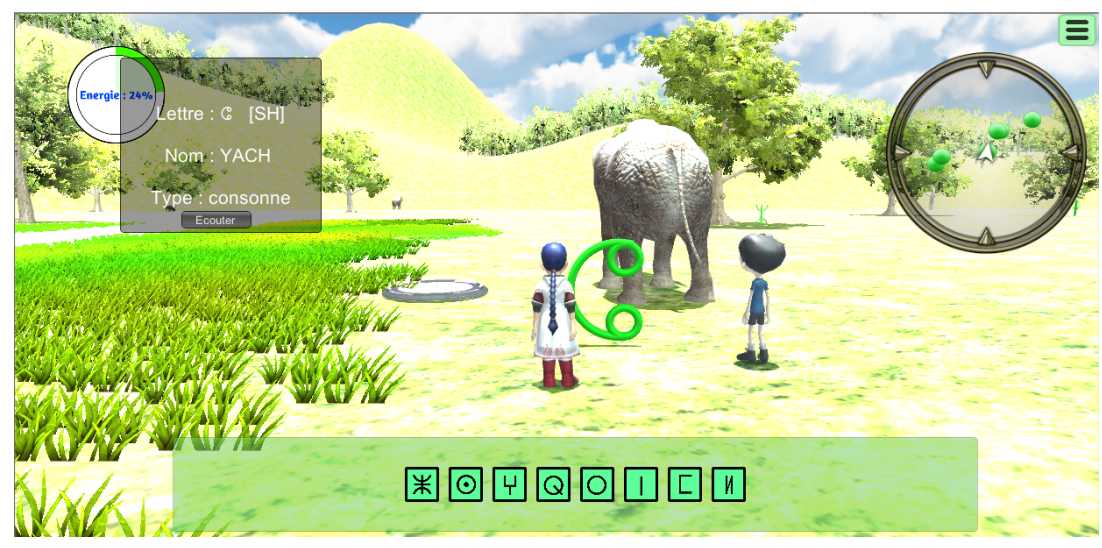

Fig. 1. Screen shot of a learning situation

\subsection{Immersive learning}

The use of the 3D environment provided by unity engine allows ImALeG project to provide immersive learning. During a game session, the flow of the learning practice is generated by the learner, and explicitly by his position and the actions he achieves (e.g. which letter he touches) in the game world.

Musical immersion is an essential game element. The player interactions, the communication of ideas and information provided must be flexible and responsive to the player actions. ImALeG contains a first music layer which is calm in order to create a perceptible atmosphere. Furthermore, it has an agitated second music layer at the time of an important event in order to give instructions and information during an element discovery.

To improve player engagement and cognitive immersion, ImaLeG provide a simple avatar control and flexible camera movement in which players use interactive mechanisms acquired in real life to accomplish artificial quests. The player actions can be decomposed into serval basic virtual behaviors grouped into four types: observe, move act with game objects and communicate with Non-player characters.

\subsection{Multi-agent concept}

To guarantee flexibility and autonomy of the system, we have chosen to create a multi-agent system based on ML-agent to control Non-Player characters (NPCS) during the scene and to track the player and to collect information that are stored in a specific database.

The agents are always enabled during the game session. Each agent executes a specific function, and they have in common a main mission of gathering data related to the evolution of the learner, treating and evaluating the data to provide an adaptive interface. Hence, we have defined the following agents as: 


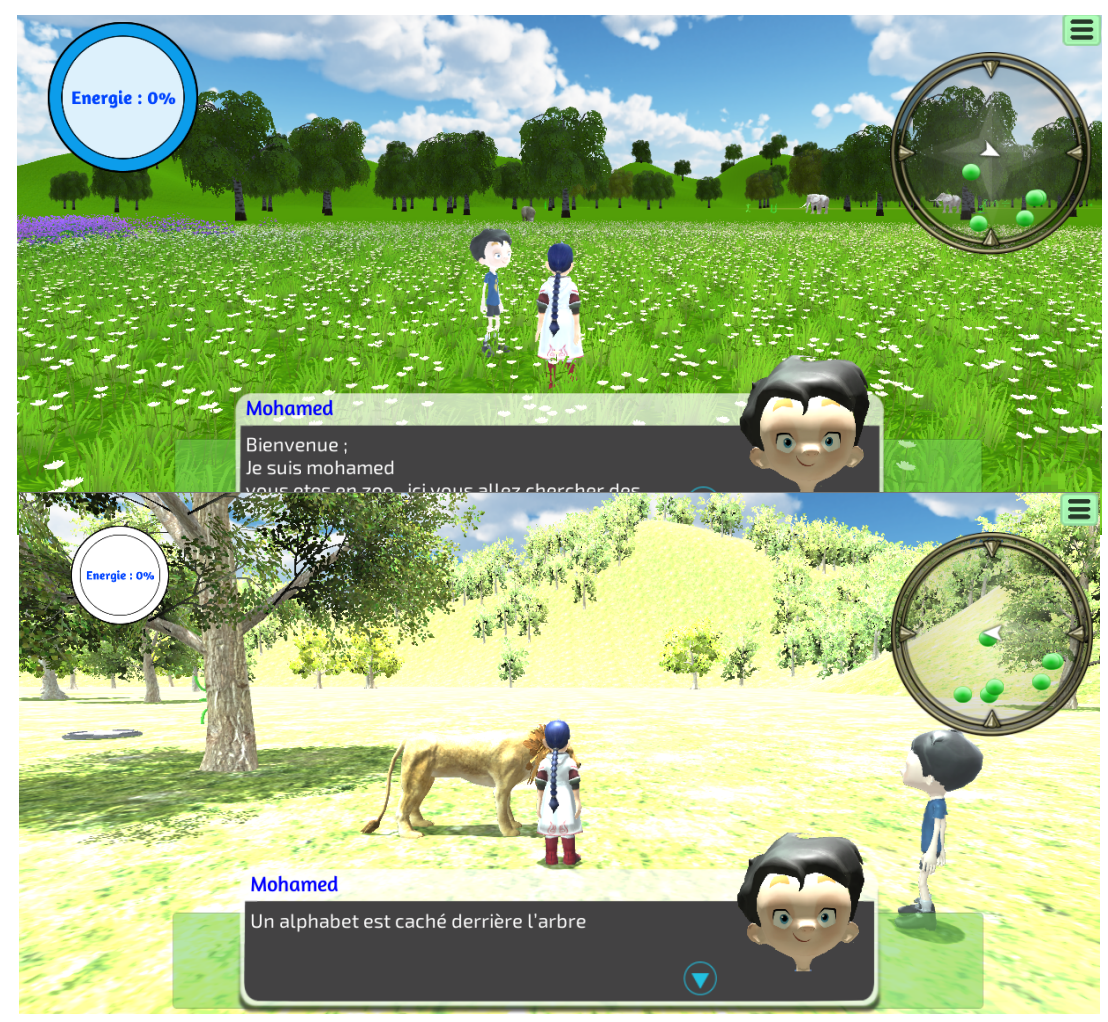

Fig. 2. Tifinaghe learning environment: "Tifinaghe world"

Tutor agent: This agent is responsible for the non-player character movement control and the detection of learning situations that reach dialog agent.

Collect Agent: Collect agent responsible for gathering data related to the player evolution in the virtual environment (scores obtained, the correct answers, errors). He sends this information to the database to keep tracking the learning evolution.

Dialogue Agent: The dialogue agent is responsible for loading dialogues data for the player and provides them to other agents or to the environment.

\subsection{Scoring}

In addition to scoring which helps to" keep" the learner playing, the learner must full the health bar. Then, he will have the necessary energy that gives him the right to be teleported to another level. The scoring of the different learning activities is employed to build the learner model and to establish the skill level of the learner in supplementary interactions. 


\subsection{Evaluation data}

The game has been achieved within the Unity engine environment. The purpose for selecting unity engine environment is twofold. Firstly, it offers high level 3D graphical tools thus helping the design of virtual worlds. Secondly, being available by everyone over the Internet, Unity engine allows collecting large amounts of test data for evaluation and player's progress tracking.

\subsection{Game architecture}

Our game communicates with the databases using network manager of the game engine that offer the player the possibility to become a server and client at the same time and to send collected information in real time through an API System.

Collect agent handles the collection of scores and information that come from the game scene and other agents and stores the results information corresponding to score obtained, number of errors made in each level and number of feedbacks that took place to resolve the quest.

The tutor agent allows control over the Non-player character (NPC tutor) and load and display conversation that comes from the dialogue agent.

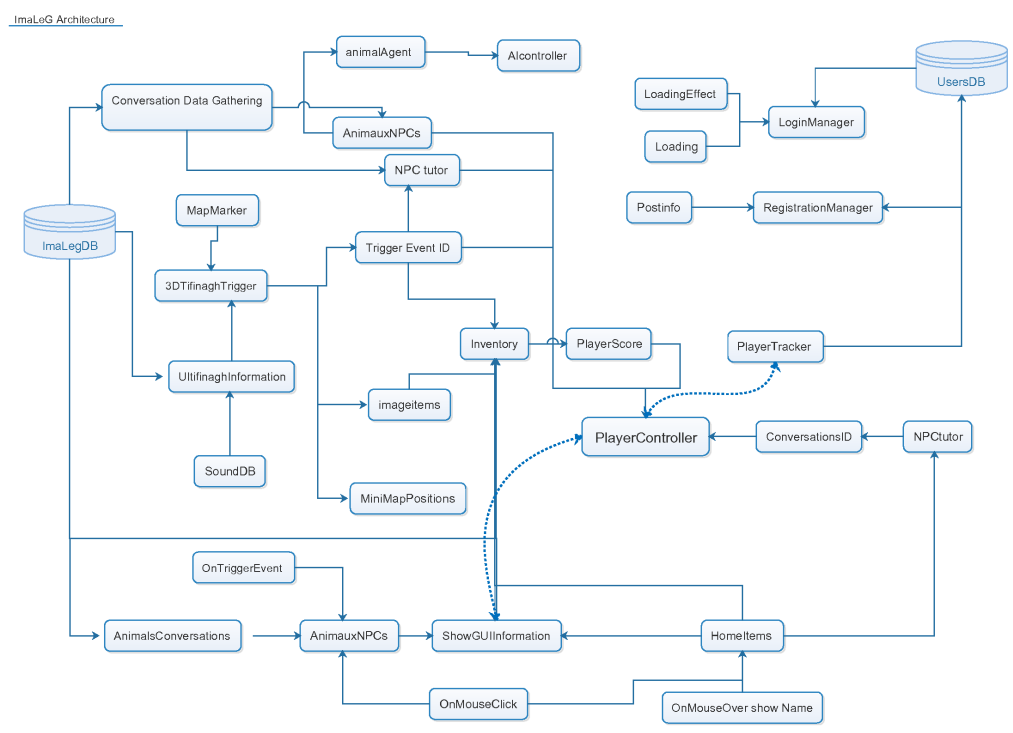

Fig. 3. ImALeG Game architecture

\section{Game Evaluation}

In order to evaluate ImALeG, we have tested our game on groups with different age and discipline. At the end of each game session, players answer a survey available 
on google form which has four parts of question, each one deals a part of our proposed assessment approach:

Immersion, manipulation and accessibility:

- Understanding the interaction modes

- Define players feeling towards the avatar, Ease of navigation in the scene

\section{Skills acquisition}

- If the arguments, actions, communication modes, methods, strategies used in the game useful for each learning situation

- If the player is able to identify each Tifinaghe alphabets

\section{Balance between challenges and rewards}

- Define if there any reward for each quest of the game that requires intellectual effort

- Moments witch the player felt lack of motivation

\section{Game Mechanics}

- Arguments, actions, communication, methods and strategies allowed the player to evolve in the game

- Strategy and actions used to resolve quests

We chose two players groups; the first group contain players in age between 10 and 18 years old and another group contain players with age between 18 and 28 years old. The result obtained left a positive impact that encourages us to progress in our work and broadens the competencies scope.

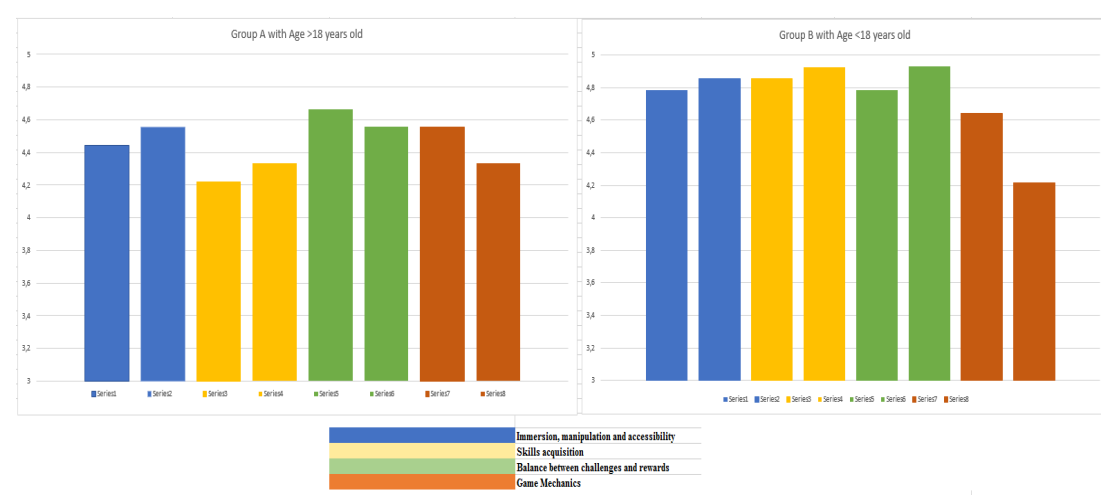

Fig. 4. Evaluation result diagram

Players carry answers between 1 and 5 which indicates the degree of satisfaction on one of the game entities. We calculate the average of answers from each group of questions; we illustrate the result for both groups of players (Fig.4). 


\section{Discussion}

The diagram obtained shows the degree of satisfaction, immersion, ease of manipulation, challenge, and the acquisition of skills for both age groups. Indicating the greater desire of both groups but mostly group to participate in another event of this kind compared to Group A. In addition, Group B is much more open to new learning methods after the event, highlighting satisfaction with the Serious Game. the study showed that ImaLeG achieved better scores in all categories considered (learning success, motivation, fun, satisfaction) compared to the frontal lessons. Thus, this serious game is well suited to teach learning content and thereby generate increased language learning activities. It was fun for students to gather energy and obtain abilities to teleport which increased active participation and immersion and students' skill acquisition in both groups.

ImALeG positively influenced the learning success that could be confirmed. The motivation and the fun factor were in the Serious Game significantly higher than in the frontal lessons. The evaluation survey showed that the students were visibly more satisfied with the serious game approach compared to the frontal teaching method. We found from the analysis of the results that players under 18 years old present a greater degree of satisfaction and skills acquisition, although that ImaLeG suitable for all age groups who want to learn Tifinaghe and Amazighe language this tiny difference reflects the children tendency to video games.

\section{Conclusion}

This paper describes a serious game that supports the acquisition of the principal knowledge and skills that are necessary in Amazigh language. Being a game has the advantage of being much more affordable and entertaining than other traditional teaching. The game is based on the immersive scenario in which a project is developed. The player must be able to develop all the phases that make up each module. Currently, the tool is being evaluated by experts through a quality model for serious games. After the improvements provided by experts who are evaluating the game as interactive 3D language game for learning Tifinaghe alphabet for Amazigh language. Our game makes use of virtual environment technology allowing immersive language learning and exploits multi-agent system to provide autonomy. We will as a future work extends ImALeG by taking into account vocabulary language. We further plan to formalize the process of evaluation of the learner output, and to define more proficient behavior to present feedbacks to learners.

\section{References}

[1] Telmem, M., \&Ghanou, Y. (2017, October). Amazigh Speech Recognition System Based on CMUSphinx. In Proceedings of the Mediterranean Symposium on Smart City Applications (pp. 397-410). Springer, Cham. https://doi.org/10.1007/978-3-319-74500-8 37 
[2] Talha, M., Boulaknadel, S., \& Aboutajdine, D. (2018, February). Performance Evaluation of SVM-Based Amazighe Named Entity Recognition. In International Conference on Advanced Machine Learning Technologies and Applications (pp. 232-241). Springer, Cham. http://doi.org/10.1007/978-3-319-74690-6 23

[3] Lu, A., Chan, S., Cai, Y., Huang, L., Nay, Z. T., \& Goei, S. L. (2018). Learning through VR gaming with virtual pink dolphins for children with ASD. Interactive Learning Environments, 26(6), 718-729. http://doi.org/10.1080/10494820.2017.1399149

[4] Yedri, O. B., El Aachak, L., Belahbib, A., Zili, H., \& Bouhorma, M. (2017, October). Learners' motivation analysis in serious games. In Proceedings of the Mediterranean Symposium on Smart City Applications (pp. 710-723). Springer, Cham. https://doi.org/10.10 07/978-3-319-74500-8 65

[5] M. Dunne. SGLL Project X. Dublin Institute of Technology, Dublin, Ireland,2008.

[6] T. Vogel, S. Cook. “MiddWorld Online.” Middlebury College, Middlebury, VT,2010.

[7] J. Westra, F. Dignum,V.Dignum, Guiding user adaptation in serious games. In Agents for games and simulations II. Springer Berlin Heidelberg. 117-131,2011. https://doi.org/10.10 07/978-3-642-18181-8 9

[8] M. Bratman,E."Intentions, Plans, and Practical Reason.” Harvard University Press, Cambridge, MA.1987.

[9] D. Cohen, Chamito-sémitiques (langues). In EncyclopædiaUniversalis, 2007.

[10] Casañ-Pitarch, R. (2018). Smartphone Serious Games as a Motivating Resource in the LSP Classroom. Journal of ForeignLanguage Education and Technology, 3, 2.

[11] G. Delmas, R. Champagnat, M. Augeraud,"Plot monitoring for interactive narrative games." In Proceedings of the international conference on Advances in computer entertainment technology. 17-20, 2007. https://doi.org/10.1145/1255047.1255051

[12] S. Alvarez-Napagao, F. Koch, I. Gómez-Sebastià,J. Vázquez-Salceda,“Making games alive: an organisational approach. In Agents for games and simulations II." 179-191. Springer Berlin Heidelberg, 2011. https://doi.org/10.1007/978-3-642-18181-8 13

[13] Bruce, K.B., Cardelli, L., Pierce, B.C.: Comparing Object Encodings. In: Abadi, M., Ito, T. (eds.): Theoretical Aspects of Computer Software. Lecture Notes in Computer Science, Vol. 1281. Springer-Verlag, Berlin Heidelberg New York (1997) 415-438. https://doi.org/ $\underline{10.1007 / \mathrm{BFb} 0014561}$

[14] Yang, H., Ma, W., Gong, D., Hu, J., \& Yao, D. (2014). A longitudinal study on children's music training experience and academic development. Scientific reports, 4, 5854. https://doi.org/10.1038/srep05854

[15] Nejme, F. Z., Boulaknadel, S., \& Aboutajdine, D. (2012). Toward an amazigh language processing. In Proceedings of the 3rd Workshop on South and Southeast Asian Natural Language Processing (pp. 173-180).

[16] Satori, H., \& ElHaoussi, F. (2014). Investigation Amazigh speech recognition using CMU tools. International Journal of Speech Technology, 17(3), 235-243. https://doi.org/10.1007/ s10772-014-9223-y

[17] Elboubekri, A. (2017). The Intercultural Communicative Competence and Digital Education: The Case of Moroccan University Students of English in Oujda. Journal of Educational Technology Systems, 45(4), 520-545. https://doi.org/10.1177/0047239516670 $\underline{994}$ 


\section{Authors}

Yassine Tazouti Is a PhD student of serious game design and development at the Laboratory of Research in computer science and Telecommunications (LaRIT) faculty of science kénitra, Morocco. He was awarded a master of mathematics and information technology from Abdelmalek Essaadi university, Tetouan-Morocco in 2014.

Siham Boulaknadel Is a researcher at the Royal Institute of Amazighe Culture, Associate Researcher at the Laboratory of Research in Computer Science and Telecommunications (LRIT) Faculty of Sciences of Rabat and Member of the Skill Pole STIC. His research interests include automatic language processing, information retrieval, artificial intelligence and distance learning.

Youssef Fakhri Received his Bachelor's Degree (B.S) in Electronic Physics in 2001 and his Master's Degree (DESA) in Computer and Telecommunication from the Faculty of Sciences, University Mohammed V, Rabat, Morocco, in 2003 where he developed his Master's Project at the ICI Company, Morocco. He received a PhD in 2007 from the University Mohammed V - Agdal, Rabat, Morocco in collaboration with the Polytechnic University of Catalonia (UPC), Spain. He joined the Faculty of Sciences of Kénitra, Department of Computer Science and Mathematics, IbnTofail University, Morocco, as an Associate Professor in March 2009, he is the Laboratory head at LaRIT (Laboratory for Research in Computing and Telecommunications) in the Faculty of Kénitra, and Member of Pole of Competences STIC Morocco.

Article submitted 2019-05-13. Resubmitted 2019-07-08. Final acceptance 2019-07-09. Final version published as submitted by the authors. 